\title{
Energy of the quasi-free electron in xenon
}

\author{
Xianbo Shi ${ }^{a}$, Luxi Li $^{\text {a }}$, C.M. Evans ${ }^{\text {a,* }}$, G.L. Findley ${ }^{\mathrm{b}}$ \\ ${ }^{a}$ Department of Chemistry and Biochemistry, Queens College - CUNY, Flushing, \\ NY 11367, United States and Department of Chemistry, Graduate Center - \\ CUNY, New York, NY 10016, United States \\ ${ }^{\mathrm{b}}$ Department of Chemistry, University of Louisiana at Monroe, Monroe, LA \\ 71209, United States
}

\begin{abstract}
Field ionization of trimethylamine and of N,N-dimethylaniline doped into xenon is presented as a function of xenon number density up to the density of the triple point liquid, both at noncritical temperatures and along the critical isotherm. These data exhibit a decrease in the xenon induced shift of the dopant ionization energy near the xenon critical point. The energy of the quasi-free electron, arising from dopant field ionization, in xenon is calculated within a local Wigner-Seitz model to within $\pm 0.3 \%$ of experiment at noncritical temperatures and for the critical isotherm.
\end{abstract}

Key words: supercritical xenon, field ionization, quasi-free electron energy, electron mobility

PACS: 33.15.Ry, 34.30.+h, 31.70.-f, 31.70.Dk

\section{Introduction}

Because of its chemical inertness and its relatively high critical temperature and low critical pressure, xenon is a popular supercritical solvent for probing density effects on electron mobility in highly polarizable fluids (see, for example, $[1-6]$ ) and for investigating pulse radiolysis reaction kinetics (see, for example, [6-8]). While xenon critical effects on electron mobility have been explored $[9,10]$, an investigation of these effects on the quasi-free electron energy

* Corresponding author.

Email addresses: xshi@gc.cuny.edu (Xianbo Shi), lli1@gc.cuny.edu (Luxi Li), cherice.evans@qc. cuny.edu (C.M. Evans), findley@ulm.edu (G.L. Findley). 
in xenon has not been performed. We have recently shown that the quasi-free electron energy $V_{0}\left(\rho_{\mathrm{P}}\right)$ in argon [11,12] and in krypton [13] increases significantly near the critical density and temperature of the perturber. We were able to predict to within $\leq \pm 0.2 \%$ of experiment this change in $V_{0}\left(\rho_{\mathrm{P}}\right)$ along the critical isotherm [11-13], as well as the overall perturber-dependent energy shift in $V_{0}\left(\rho_{\mathrm{P}}\right)$ [14] from low perturber density to the density of the triple point liquid, using a novel local Wigner-Seitz model [11-14] for the quasi-free electron energy. The success of this local Wigner-Seitz model in predicting the critical isotherm changes in $V_{0}\left(\rho_{\mathrm{P}}\right)$ indicated that the variations observed arise from fluctuations in the local density of perturber atoms surrounding the quasi-free electron.

In this paper, we report the xenon induced shift $\Delta_{\mathrm{D}}\left(\rho_{\mathrm{Xe}}\right)$ of the dopant ionization energies for trimethylamine and $N, N$-dimethylaniline at various xenon number densities $\rho_{\mathrm{x}}$ up to the density of the triple point liquid, both at noncritical temperatures and near the critical isotherm of xenon. We show that $\Delta_{\mathrm{D}}\left(\rho_{\mathrm{Xe}}\right)$ decreases along the critical isotherm near the critical density of xenon. From these data, we have extracted the energy $V_{0}\left(\rho_{\mathrm{xe}}\right)$ of the quasi-free electron in xenon using [11-15]

$$
V_{0}\left(\rho_{\mathrm{P}}\right)=\Delta_{\mathrm{D}}\left(\rho_{\mathrm{P}}\right)-P_{+}\left(\rho_{\mathrm{P}}\right),
$$

where $P_{+}\left(\rho_{\mathrm{Xe}}\right)$ is the ensemble average polarization energy of xenon by the dopant ionic core. We show that the dopant dependence in $\Delta_{\mathrm{D}}\left(\rho_{\mathrm{xe}}\right)$ can be completely accounted for by $P_{+}\left(\rho_{\mathrm{xe}}\right)$ with the appropriate choice of intermolecular potential parameters. We then accurately reproduce (to within $\pm 0.3 \%$ of experiment) the quasi-free electron energy $V_{0}\left(\rho_{\text {xe }}\right)$ using the local Wigner-Seitz model [11-14].

\section{Experimental}

Trimethylamine (Matheson Gas Products, 99.5\%), N,N-dimethylaniline (Aldrich, 99.8\%) and xenon (Matheson Gas Products, 99.995\%) were used without further purification. The absence of trace impurities in the spectral range of interest was verified by the measurement of photoabsorption spectra of all chemicals in this study. The number density of xenon was calculated from the Strobridge equation of state for $\mathrm{N}_{2}$ [16], with the Xe coefficients obtained from Streett, et al. [17], using a standard iterative technique. Both the gas handling system and the procedures to ensure homogeneous mixing of the dopant and perturber have been described previously [11-14,18]. Prior to the introduction of any dopant/perturber system, the experimental cell and gas handling system were baked to a base pressure of $10^{-8}$ Torr, and in order to ensure no perturber contamination by the dopant (which was present at a concentration 
of $<10 \mathrm{ppm}$ ) after the addition of the dopant, the gas handling system was allowed to return to the low $10^{-7}$ Torr range before the addition of xenon.

Photoionization spectra were measured with monochromatized synchrotron radiation [18] having a resolution of $1.8 \AA$ (or $8 \mathrm{meV}$ in the spectral region of interest). The copper experimental cell, capable of withstanding pressures of up to 100 bar, is equipped with entrance and exit $\mathrm{MgF}_{2}$ windows $(1 \mathrm{~cm}$ path length) and a pair of parallel plate electrodes (stainless steel, $3 \mathrm{~mm}$ spacing) oriented perpendicular to the windows [18]. This experimental cell is attached to an open flow liquid $\mathrm{N}_{2}$ cryostat and resistive heater that allowed the temperature to be controlled to within $\pm 0.3^{\circ} \mathrm{C}$ at noncritical temperatures and to within $\pm 0.1^{\circ} \mathrm{C}$ at temperatures near the critical isotherm. The measurement of dopant photoionization spectra at noncritical temperatures was performed at $19.8^{\circ} \mathrm{C}$ for xenon densities of $(0.5-6.3) \times 10^{21} \mathrm{~cm}^{-3}$ and at $18.0^{\circ} \mathrm{C}$ for xenon densities of $(6.4-7.0) \times 10^{21} \mathrm{~cm}^{-3}$. Below the critical temperature, the xenon isotherms are very straight, implying a small density change over a large pressure range at constant temperature. Therefore, in the density region of $(7.5-13.6) \times 10^{21} \mathrm{~cm}^{-3}$, the measurement temperature was selected based on the desired number density, with the temperature ranging from $13.0^{\circ} \mathrm{C}$ for the xenon density of $7.5 \times 10^{21} \mathrm{~cm}^{-3}$ to $-109.5^{\circ} \mathrm{C}$ for the xenon density of $13.6 \times 10^{21} \mathrm{~cm}^{-3}$. To prevent liquid formation in the cell during temperature stabilization, the set point for the critical isotherm was chosen to be $17.0^{\circ} \mathrm{C}$, near the xenon critical temperature of $16.5^{\circ} \mathrm{C}$. The narrowness of the phase diagram in the saddle region near the critical density $\left(\rho_{c}=5.0 \times 10^{21} \mathrm{~cm}^{-3}\right)$, which requires a $2.0 \times 10^{21} \mathrm{~cm}^{-3}$ change in density for a 1 mbar change in pressure, prohibited the measurement of photoionization in the density range $(4.5-6.5) \times 10^{21} \mathrm{~cm}^{-3}$. Saturated absorption of the Xe $6 \mathrm{~s}$ Rydberg state at high xenon number density $\rho_{\text {xe }}$ prevented the measurement of TMA photoionization spectra in xenon densities of $(0.5-3.5) \times 10^{21} \mathrm{~cm}^{-3}$. The intensity of the synchrotron radiation exiting the monochromator was monitored by measuring the current across a Ni mesh intercepting the beam prior to the experimental cell. All photoionization spectra were normalized to this current.

A field ionization spectrum of a dopant is obtained by subtracting a photoionization spectrum measured at a low field $F_{L}$ from a spectrum measured at a high field $F_{H}$ after intensity normalization [19]. The field ionization spectrum results from high- $n$ Rydberg states that are field-ionized by $F_{H}$ but not $F_{L}$. Variation in the strength of $F_{H}$ leads to a shift in the energetic position of the field ionization peak. Therefore, the xenon dependent shift $\Delta_{\mathrm{D}}\left(\rho_{\mathrm{Xe}}\right)$ of the dopant ionization energy is obtained from $[14,19]$

$$
\Delta_{\mathrm{D}}\left(\rho_{\mathrm{Xe}}\right)=I_{F}\left(\rho_{\mathrm{Xe}}\right)+c_{D}\left(\sqrt{F_{L}}+\sqrt{F_{H}}\right)-I_{g}
$$

where $I_{g}$ is the gas phase dopant ionization energy, $I_{F}\left(\rho_{\text {xe }}\right)$ is the energy of the 
field ionization peak (reflecting the dopant ionization energy perturbed by the electric field and by dense xenon), and $c_{D}$ is a dopant dependent, perturber independent field ionization constant. For this study, $I_{g}$ was evaluated using field ionization of the gas phase dopants and was determined to be $8.462 \pm 0.008$ $\mathrm{eV}$ for TMA and 7.450 $\pm 0.010 \mathrm{eV}$ for DMAn. The field ionization constant $c_{D}$ was also obtained from the gas phase dopant field ionization studies, but was verified by checking the field dependence of the dopant ionization potential at specific xenon number densities for each dopant. From these measurements, $c_{D}=2.78 \pm 0.15 \times 10^{-4} \mathrm{eV} \mathrm{cm}^{1 / 2} \mathrm{~V}^{-1 / 2}$ for TMA and $c_{D}=5.11 \pm 0.80 \times 10^{-5}$ $\mathrm{eV} \mathrm{cm}{ }^{1 / 2} \mathrm{~V}^{-1 / 2}$ for DMAn. For all field ionization measurements reported here, $F_{L}=5000 \mathrm{~V} \mathrm{~cm}^{-1}$ and $F_{H}=11,667 \mathrm{~V} \mathrm{~cm}^{-1}$. The total error range for any experimental point is given by a sum of the field correction error, the goodness-of-fit error (for fitting a field ionization spectrum to a gaussian lineshape), and the error arising from the energy uncertainty due to the resolution of the monochromator (i.e., $\pm 4 \mathrm{meV}$ ). For measurements at noncritical temperatures, this total error averages to $\pm 0.015 \mathrm{eV}$ for TMA and $\pm 0.020 \mathrm{eV}$ for DMAn. For measurements along the critical isotherm of xenon, this total error averages to $\pm 0.025 \mathrm{eV}$ for TMA and $\pm 0.030 \mathrm{eV}$ for DMAn.

\section{Results and Discussion}

The xenon induced shift of the TMA ionization energy $\Delta_{\mathrm{TMA}}\left(\rho_{\mathrm{Xe}}\right)$ is presented in Fig. 1a, and that for DMAn $\Delta_{\text {DMAn }}\left(\rho_{\text {Xe }}\right)$ is presented in Fig. 1b, for noncritical temperatures (solid markers) and for an isotherm near the critical isotherm (open markers). These data show a clear decrease in the density dependent shift of $\Delta_{\mathrm{D}}\left(\rho_{\mathrm{Xe}}\right)$ near the xenon critical density $\left(\rho_{c}=5.0 \times 10^{21} \mathrm{~cm}^{-3}\right)$. Determining the quasi-free electron energy $V_{0}\left(\rho_{\mathrm{xe}}\right)$ for xenon from these data using Eq. (1), however, requires accurate modeling of the average ion/perturber polarization energy $P_{+}\left(\rho_{\mathrm{xe}}\right)$ for each dopant/perturber system.

$P_{+}\left(\rho_{\mathrm{P}}\right)$ was calculated using $[11-14,19]$

$$
P_{+}\left(\rho_{\mathrm{P}}\right)=-4 \pi \rho_{\mathrm{P}} \int_{0}^{\infty} g_{\mathrm{PD}}(r) w_{+}(r) r^{2} d r,
$$

where $g_{\mathrm{PD}}(r)$ is the perturber/dopant radial distribution function and $w_{+}(r)$ is the perturber/ion interaction potential $[11-14,19]$. Since $w_{+}(r)$ incorporates induced dipole interactions in the perturbing medium, the perturber/perturber radial distribution function $g_{\mathrm{PP}}(r)$ is also involved in determining $P_{+}\left(\rho_{\mathrm{P}}\right)$. As was the case in our previous studies of rare gas perturbers [11-14], the radial distribution functions $g_{\mathrm{PD}}(r)$ and $g_{\mathrm{PP}}(r)$ were computed from the coupled Percus-Yevick integro-differential equation method [14,20], with a LennardJones 6-12 potential employed for the Xe/Xe interaction and a modified Stockmeyer potential written in Lennard-Jones 6-12 form [14] employed for the 

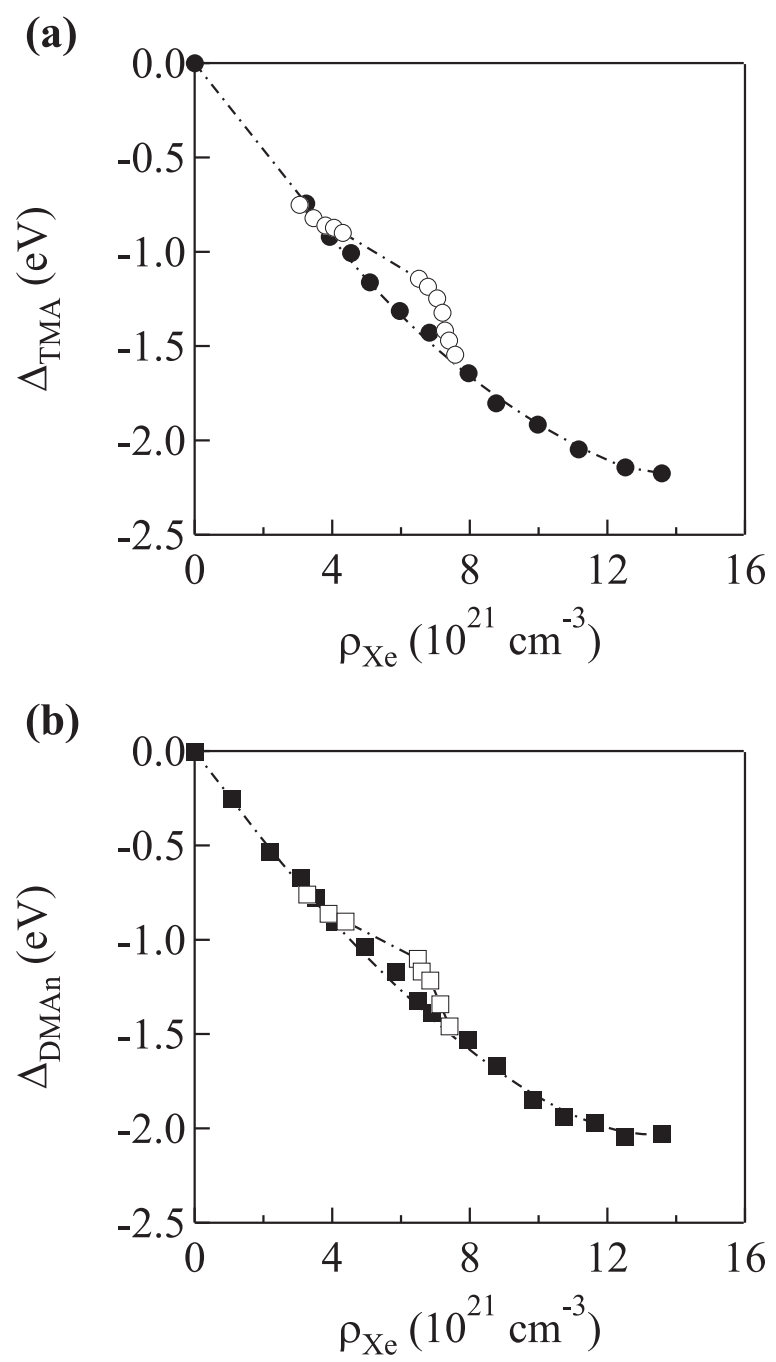

Fig. 1. Xenon induced shift of the ionization energy of (a) TMA $\Delta_{\text {TMA }}\left(\rho_{\text {Xe }}\right)$ and (b) DMAn $\Delta_{\text {DMAn }}\left(\rho_{\mathrm{Xe}}\right)$ plotted as a function of xenon number density $\rho_{\mathrm{Xe}}$ at various (see Section 2 for details) noncritical temperatures $(\bullet, \mathbf{\square})$ and for an isotherm $\left(17^{\circ} \mathrm{C}\right)$ near the xenon critical isotherm $(\circ, \square)$. The lines are provided as a visual aid.

TMA/Xe and DMAn/Xe interactions. (The Lennard-Jones 6-12 parameters were $\sigma_{\mathrm{PP}}=4.055 \AA$ and $\varepsilon_{\mathrm{PP}} / k_{B}=229.0 \mathrm{~K}$ for the Xe/Xe potential [21], $\sigma_{\mathrm{DP}}=$ $4.430 \AA$ and $\varepsilon_{\mathrm{DP}} / k_{B}=223.0 \mathrm{~K}$ for the TMA $/$ Xe potential, and $\sigma_{\mathrm{DP}}=4.950 \AA$ and $\varepsilon_{\mathrm{DP}} / k_{B}=245.0 \mathrm{~K}$ for the DMAn/Xe potential ( $k_{B} \equiv$ Boltzmann's constant). $)^{1}$ Subtracting $P_{+}\left(\rho_{\mathrm{P}}\right)$ from $\Delta_{\mathrm{D}}\left(\rho_{\mathrm{P}}\right)$ leads to the experimental values of $V_{0}\left(\rho_{\mathrm{Xe}}\right)$, which are presented in Fig. 2a.

1 The TMA/TMA and DMAn/DMAn intermolecular potential parameters were determined from the TMA and DMAn critical point data, respectively [22]. These parameters were then combined with the Xe/Xe intermolecular potential parameters using the Sikora combining rule [23], before being folded into the modified Stockmeyer potential [14]. Small adjustments $(< \pm 5 \%)$ were made to the intermolecular potential parameters to remove all dopant dependence from $V_{0}\left(\rho_{\mathrm{Xe}}\right)$. 


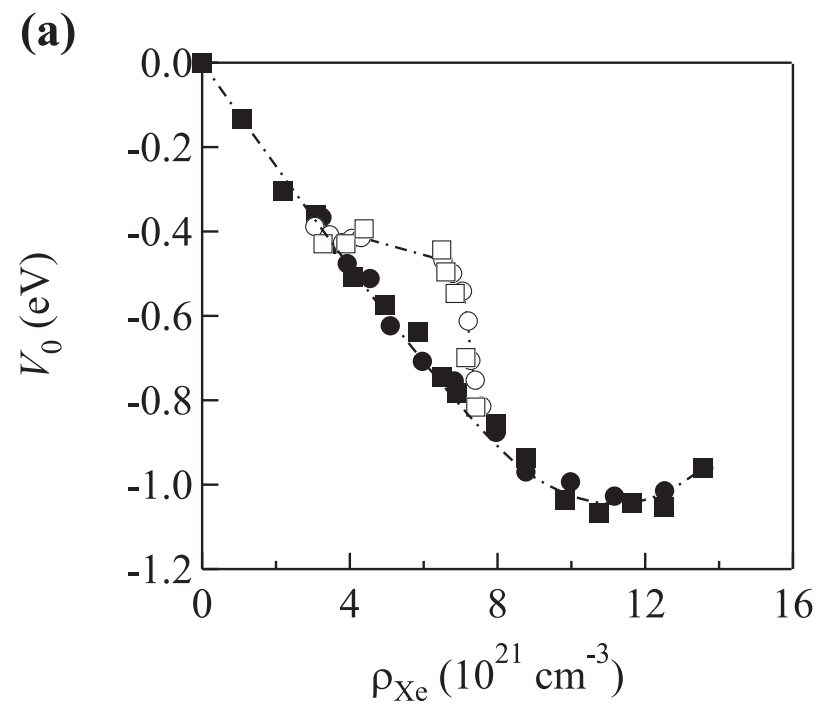

(b)

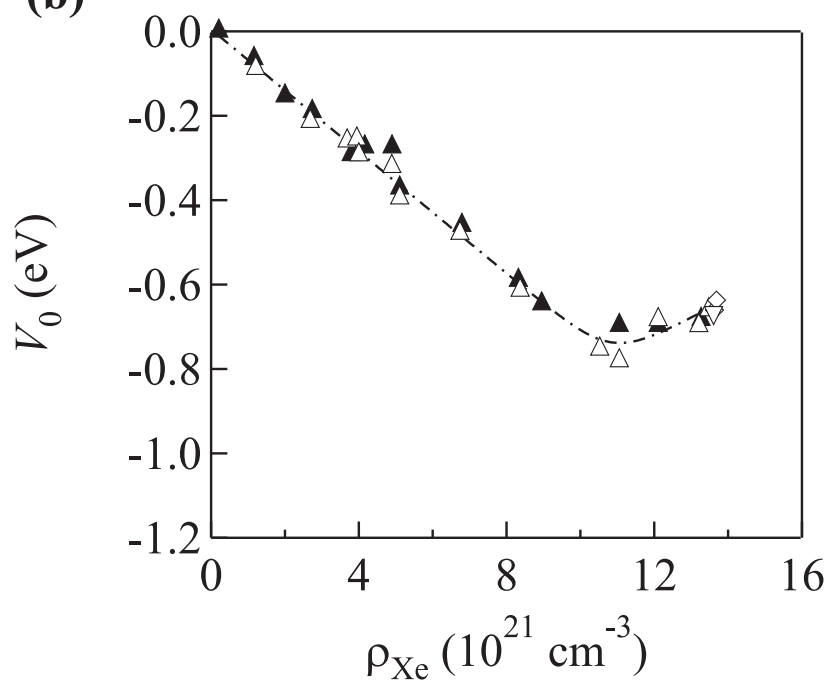

Fig. 2. (a) $V_{0}\left(\rho_{\mathrm{Xe}}\right)$ [Eq. (1)] determined by subtracting $P_{+}\left(\rho_{\mathrm{Xe}}\right)$ [Eq. (2)] from the experimentally determined [Fig. 1] xenon induced shifts of the TMA and DMAn ionization energies $\Delta_{\mathrm{TMA}}\left(\rho_{\mathrm{Xe}}\right)$ and $\Delta_{\mathrm{DMAn}}\left(\rho_{\mathrm{Xe}}\right)$, respectively, plotted as a function of xenon number density $\rho_{\mathrm{Xe}}$ at various (see Section 2 for details) noncritical temperatures (• TMA, DMAn) and near the critical temperature of xenon (o TMA, $\square$ DMAn). (b) $V_{0}\left(\rho_{\mathrm{Xe}}\right)$ obtained from various photoinjection measurements, plotted as a function of xenon number density $\rho_{\mathrm{Xe}} \cdot(\triangle, \mathbf{\Delta})[24],(\nabla)[25,26]$ and $(\diamond)[27]$. The lines are provided as a visual aid.

For purposes of comparison, previous determinations of the noncritical isotherm $V_{0}\left(\rho_{\mathrm{Xe}}\right)$ from photoinjection measurements [24-27] are presented in Fig. $2 \mathrm{~b}$. Our measurements clearly have less scatter and a larger overall shift, although the minimum in $V_{0}\left(\rho_{\mathrm{Xe}}\right)$ is located at the same xenon number density. (Moreover, the present data overlap with those obtained from the field ionization of dimethylsulfide in high density xenon $\left(\rho_{\mathrm{Xe}}>6 \times 10^{21} \mathrm{~cm}^{-3}\right)$ at noncritical temperatures [28] when appropriate dimethylsulfide/xenon intermolecular 
potential parameters are selected for the calculation of $P_{+}\left(\rho_{\mathrm{Xe}}\right)$.) The consistently smaller quasi-free electron energies $V_{0}\left(\rho_{\mathrm{P}}\right)$ obtained from photoinjection studies in the rare gases $[14,19,28]$ arise from difficulties in correcting the systematic energy errors caused by the density dependent adsorption of perturber molecules onto the photoinjection electrode surface, and by surface charging effects at the cathode. The precision exhibited in $V_{0}\left(\rho_{\mathrm{Xe}}\right)$ extracted from field ionization studies, however, allows one to see clearly the striking effect due to the critical point.

In the local Wigner-Seitz model [11-14], a one-electron Schrödinger equation with a spherically symmetric potential $V(r)$ is used to describe the interaction between the quasi-free electron and the neat fluid. However, although an average translational symmetry (neglecting fluctuations) of the potential is assumed, this translational symmetry is not presumed to be uniform. This nonuniformity is reflected by replacing the bulk number density $\rho_{\mathrm{P}}$ in the Wigner-Seitz cell [15] with a local number density defined via [11-14,29,30]

$$
\rho_{\mathrm{P}}(r)=g_{\mathrm{PP}}(r) \rho_{\mathrm{P}} .
$$

Since the maximum of $\rho_{\mathrm{P}}(r)$ gives the density of the first solvent shell, this value more closely reflects the actual number density in the neighborhood of any given perturber. In this case, then, the translational symmetry is defined by a local Wigner-Setiz radius

$$
r_{\ell}=\sqrt[3]{\frac{3}{4 \pi g_{\max } \rho_{\mathrm{P}}}},
$$

where $g_{\max }$ is the maximum of the radial distribution function. The local Wigner-Seitz radius $r_{\ell}$, therefore, represents one-half the average spacing between rare gas atoms in the first solvent shell. As in the Springett, Jortner and Cohen (SJC) model [15], we assume that $V(r)$ is divided into two parts: an attractive electron/perturber polarization energy $P_{-}\left(\rho_{\mathrm{P}}\right)$ and a repulsive atomic pseudopotential $V_{a}(r)$. We calculate the attractive electron/perturber polarization energy $P_{-}\left(\rho_{\mathrm{P}}\right)$ in a manner similar to that given for the ion/perturber polarization potential $P_{+}\left(\rho_{\mathrm{P}}\right)$ in Eq. (2), but with an interaction potential $w_{-}(r)$ originally proposed by Lekner [11,31]. Using this interaction potential, the ensemble average electron/perturber polarization energy is obtained from [11-14]

$$
P_{-}\left(\rho_{\mathrm{P}}\right)=-4 \pi \rho_{\mathrm{P}} \int_{0}^{\infty} g_{\mathrm{PP}}(r) w_{-}(r) r^{2} d r .
$$

In the evaluation of $P_{-}\left(\rho_{\mathrm{Xe}}\right)$ from Eq. (5), we again chose a Lennard-Jones 6-12 potential for the xenon/xenon interactions and, for consistency, used the same potential parameters employed in the determination of the average cation/xenon polarization energy $P_{+}\left(\rho_{\mathrm{xe}}\right)$ and the same integro-differential equation method $[14,20]$ for determining the xenon/xenon radial distribution function. The results of our calculations are presented in Fig. 3a as a function 


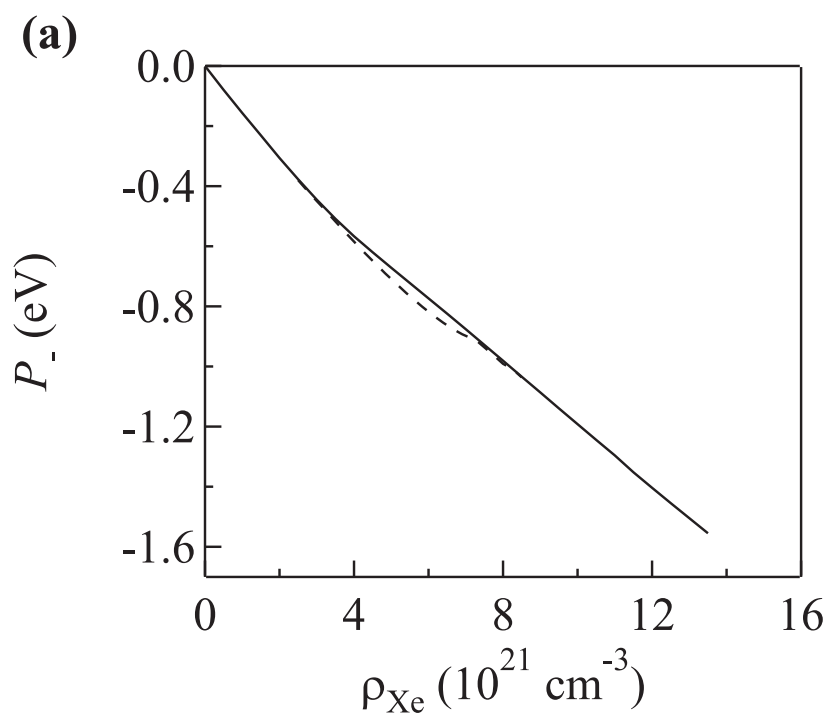

(b)

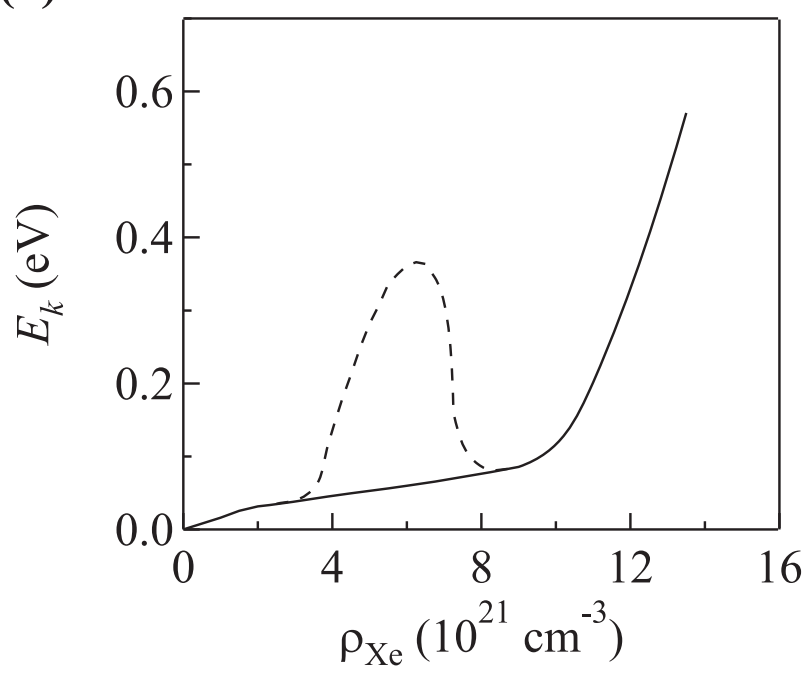

Fig. 3. Summary of the calculations necessary to model $V_{0}\left(\rho_{\mathrm{Xe}}\right)$. (a) The average electron/xenon polarization energy $P_{-}\left(\rho_{\mathrm{xe}}\right)$, and (b) the zero-point kinetic energy $E_{k}$ of the quasi-free electron, plotted as a function of xenon number density $\rho_{\mathrm{Xe}}$. The solid line is for various (see Section 2 for details) noncritical temperatures; the dashed line is for an isotherm $\left(17^{\circ} \mathrm{C}\right)$ near the critical isotherm.

of xenon number density $\rho_{\mathrm{xe}}$ and clearly show an increase in the xenon induced shift near the critical point, similar in behavior and magnitude to that observed for $P_{-}\left(\rho_{\mathrm{P}}\right)$ in $\operatorname{Ar}[11,12]$ and $\mathrm{Kr}[13]$ and for UV-vis absorption bands [32].

The potential $V(r)$ in the one-electron Schrödinger equation therefore becomes $V(r)=V_{a}(r)+P_{-}\left(\rho_{\mathrm{P}}\right)$, where $P_{-}\left(\rho_{\mathrm{P}}\right)$ is a constant for a fixed perturber number density. As in the SJC treatment [15], we define $V_{a}(r)$ as a hard-sphere potential (i.e., $V_{a}(r)=0$ for $r>r_{h}$ and $V_{a}(r)=\infty$ for $r<r_{h}$, where $r_{h}$ is the hard-sphere radius), but we set $r_{h}$ equal to the absolute value of the scattering length $A$ of the perturber. Finally, a dopant-independent phase shift 
is introduced to reflect the fact that outside the first solvent shell the quasifree electron wavefunction can also scatter off the rare gas atoms contained within the solvent shell. For $s$-wave scattering, and in the limit of small $k_{0}$, this phase shift is given by $\eta \pi$, where $\eta$ is the phase shift amplitude [11-14,33]. Incorporating this phase shift into the solution of the one-electron Schrödinger equation under the boundary conditions

$$
\psi_{0}(|A|)=0,\left.\quad\left(\frac{\partial \psi_{0}}{\partial r}\right)\right|_{r=r_{\ell}}=0
$$

yields the wavevector equation for the quasi-free electron [11-14]:

$$
\tan \left[k_{0}\left(r_{\ell}-|A|\right)+\eta \pi\right]=k_{0} r_{\ell} .
$$

In this model, $\eta$ is a perturber-dependent (but dopant-independent) parameter that is evaluated from the field ionization data for $V_{0}\left(\rho_{\mathrm{P}}\right)$ from the noncritical isotherm experiments. Once the thermal kinetic energy of the quasi-free electron is included, $V_{0}\left(\rho_{\mathrm{P}}\right)$ becomes [11-14]

$$
V_{0}\left(\rho_{\mathrm{P}}\right)=E_{k}+P_{-}\left(\rho_{\mathrm{P}}\right)+\frac{3}{2} k_{B} T, \quad E_{k}=\frac{\left(\hbar k_{0}\right)^{2}}{2 m},
$$

where $k_{0}$ is evaluated from Eq. (7). It is important to note that the density dependence of $E_{k}$ arises from the change in the local Wigner-Seitz radius as a function of the bulk number density, similar to the density dependence of $E_{k}$ in the original SJC model [15]. However, $E_{k}$ also inherits an implicit temperature dependence from the local Wigner-Seitz radius [11-13], a dependence that is absent in the SJC model [15]. Fig. 3b presents $E_{k}$ for xenon as determined from Eqs. (7)-(8), with $\eta=0.51$ and $A=-3.24 \AA$ [34], plotted as a function of xenon number density. One can clearly see the large increase in $E_{k}$ near the critical density and temperature of xenon. Similarly, $V_{0}\left(\rho_{\mathrm{xe}}\right)$ obtained from Eq. (8) is plotted in Fig. 4 in comparison to the experimentally determined values (cf. Fig. 2a), for noncritical temperatures (solid line) and for an isotherm near the critical isotherm (dashed line). The calculated $V_{0}\left(\rho_{\mathrm{Xe}}\right)$ closely matches experiment over the entire density range, with a scatter of $\leq \pm 0.3 \%$ of experiment that easily falls within the experimental error of $\sim \pm 0.030 \mathrm{eV}$. Moreover, $V_{0}\left(\rho_{\mathrm{Xe}}\right)$ shows an increase along the critical isotherm near the critical density of xenon, an increase that is reported here for the first time. (It is important to note that there is only a single adjustable parameter $\eta$ in this model, and that $\eta$ is determined using only the noncritical temperature data.)

Finally, since $E_{k}$ increases by $0.30 \mathrm{eV}$ along the critical isotherm near the critical point while $P_{-}\left(\rho_{\mathrm{xe}}\right)$ decreases by $0.04 \mathrm{eV}$, it is clear that $E_{k}$ is the determining factor for the decrease in the shift of $V_{0}\left(\rho_{\mathrm{xe}}\right)$. This increase in $E_{k}$ near the critical density on the critical isotherm is directly related to the 
(a)

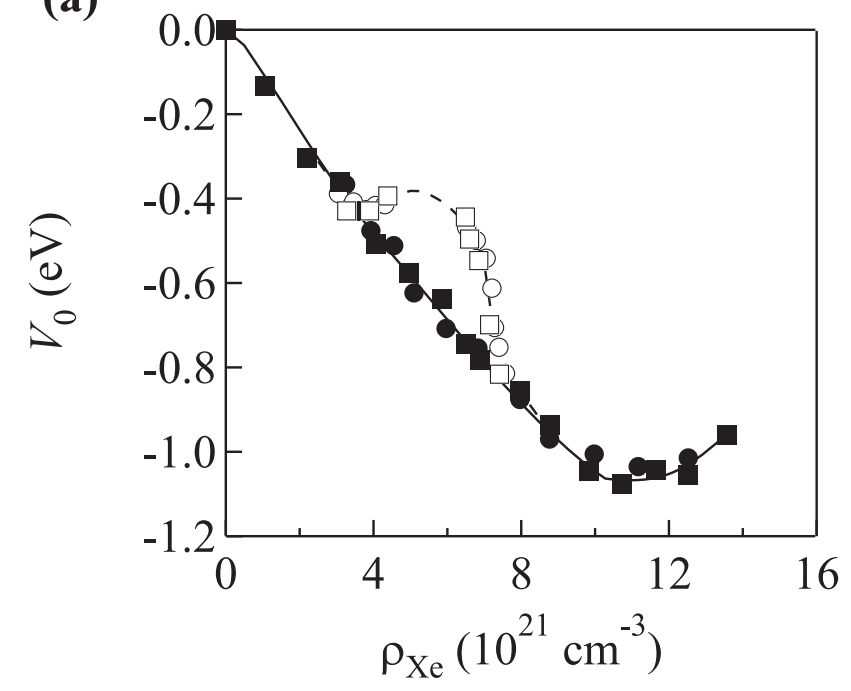

(b)

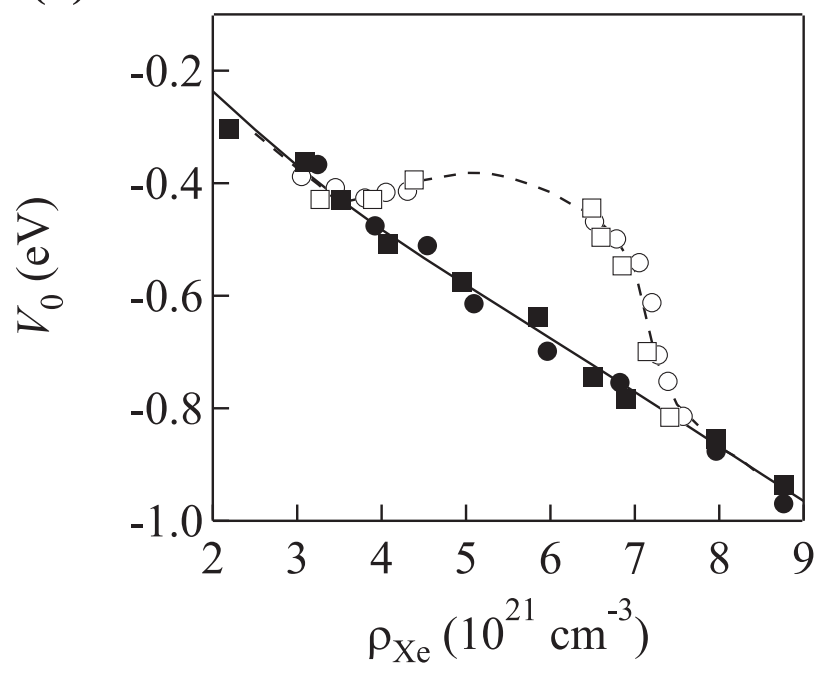

Fig. 4. Comparison of experiment (markers) with theory (lines) for the energy $V_{0}\left(\rho_{\mathrm{xe}}\right)$ of the quasi-free electron in xenon, plotted as a function of xenon number density $\rho_{\mathrm{Xe}}$, (a) for the entire density region up to the density of the triple point liquid and (b) for the critical density region. See the legend of Fig. 2a for the definition of the markers. The solid line is for various (see Section 2 for details) noncritical temperatures; the dashed line is for an isotherm $\left(17^{\circ} \mathrm{C}\right)$ near the critical isotherm.

critical point fluctuations that lead to an increase in the radial distribution function for the first solvent shell, which in turn causes a decrease in the local Wigner-Seitz radius: In other words, as the boundary condition defining the translational symmetry for the quasi-free electron wavefunction decreases, the kinetic energy of the electron must increase. Furthermore, those number densities that delimit the deviations of $E_{k}$ from the noncritical isotherm coincide with the turning points that bound the saddle point region along the critical isotherm in the xenon phase diagram. This behavior is similar to that 
recently found for argon [11,12] and krypton [13], although the increase in $E_{k}$ is nearly a factor of 4 less in argon and nearly a factor of 2 less in krypon, which presumably results from the greater polarizability of xenon.

\section{Acknowledgements}

The experimental measurements reported here were performed at the University of Wisconsin Synchrotron Radiation Center (NSF DMR-0537588). This work was supported by grants from the Petroleum Research Fund (41378-G6), from the Professional Staff Congress - City University of New York (60074-34

35 ) and from the Louisiana Board of Regents Support Fund (LEQSF(200609)-RD-A33).

\section{References}

[1] P. Stampfli, Phys. Rep. 255 (1995) 1, and references therein.

[2] R. A. Holroyd, K. Itoh, M. Nishikawa, J. Chem. Phys. 118 (2003) 706.

[3] N. A. Gorbunov, A. S. Melnikov, I. Smurov, Phys. Plasmas 10 (2003) 4552.

[4] I. T. Steinberger in Classical Rare Gas Liquids, W. F. Schmidt, E. Illenberger, editors, American Science Publishers, 2005, and references therein.

[5] S. Kobayashi, N. Hasebe, T. Igarashi, T. Miyachi, M. Miyajima, H. Okada, T. Doke, E. Shibamura, V. V. Dmitrenko, K. F. Vlasik, Jap. J. App. Phys. 43 (2004) 5568.

[6] R. A. Holroyd, J. F. Wishart, M. Nishikawa, K. Itoh, J. Phys. Chem. B 107 (2003) 7281, and references therein.

[7] R. A. Holroyd, M. Nishikawa, K. Itoh, Rad. Phys. Chem. 74 (2005) 146, and references therein.

[8] O. S. Jina, X. Z. Sun, M. W. George, Dalton Trans. (2003) 1773.

[9] T. Kimura, G. R. Freeman, J. Chem. Phys. 60 (1974) 4081.

[10] S. S. -S. Huang, G. R. Freeman, J. Chem. Phys. 68 (1978) 1355.

[11] C. M. Evans, G. L. Findley, Chem. Phys. Lett. 410 (2005) 242.

[12] C. M. Evans, G. L. Findley, J. Phys. B: At. Mol. Opt. Phys. 38 (2005) L269.

[13] Luxi Li, C. M. Evans, G. L. Findley, J. Phys. Chem. A 109 (2005) 10683.

[14] C. M. Evans, G. L. Findley, Phys. Rev. A 72 (2005) 022717. 
[15] B. E. Springett, J. Jortner, M. H. Cohen, J. Chem. Phys. 48 (1968) 2720.

[16] T. R. Strobridge, Thermodynamic Properties of Nitrogen from 114 to $540^{\circ} R$ between 1.0 and 3000 PSIA. Supplement A, U.S. Natl. Bur. Stand. Tech. Note 129 (Washington, DC, 1962).

[17] W. B. Streett, L. S. Sagan, L. A. K. Staveley, J. Chem. Thermodynamics 5 (1973) 633.

[18] C. M. Evans, J. D. Scott, G. L. Findley, Rec. Res. Dev. Chem. Phys. 3 (2002) 351.

[19] A. K. Al-Omari, Ph.D. Dissertation, University of Wisconsin Madison, Madison, WI, 1996. See also A. K. Al-Omari, K. N. Altmann, R. Reininger, J. Chem. Phys. 105 (1996) 1305.

[20] E. W. Grundke, D. Henderson, R. D. Murphy, Can. J. Phys. 51 (1973) 1216.

[21] S. V. Khristenko, A. I. Maslov, V. P. Shevelko, Molecules and Their Spectroscopic Properties, Springer-Verlag, 1998.

[22] J. J. Potoff, A. Z. Panagiotopoulos, J. Chem. Phys. 109 (1998) 10914.

[23] P. T. Sikora, J. Phys. B: At. Mol. Opt. Phys. 3 (1970) 1475.

[24] R. Reininger, U. Asaf, I. T. Steinberger, Chem. Phys. Lett. 90 (1982) 287.

[25] W. Tauchert, W. F. Schmidt, Z. Naturforsch 30A (1975) 1085.

[26] W. Tauchert, H. Jungblut, W. F. Schmidt, Can. J. Chem. 55 (1977) 1860.

[27] W. von Zdrojewski, J. G. Rabe, W. F. Schmidt, Z. Naturforsch. 35A (1980) 672 .

[28] K. N. Altmann, R. Reininger, J. Chem. Phys. 107 (1997) 1759.

[29] P. Attard, J. Chem. Phys. 91 (1989) 3072.

[30] P. Attard, J. Chem. Phys. 91 (1989) 3083.

[31] J. Lekner, Phys. Rev. 158 (1967) 130.

[32] S. C. Tucker, Chem. Rev. 99 (1999) 391, and references therein.

[33] F. Calogero, Variable Phase Approach to Potential Scattering, Academic Press, 1967.

[34] I. T. Steinberger, U. Asaf, G. Ascarelli, R. Reininger, G. Reisfeld, M. Reshotko, Phys. Rev. A 42 (1990) 3135. 